\title{
Electrostatic Interactions Govern Extreme Nascent Protein Ejection 2Times from Ribosomes and Can Delay Ribosome Recycling
}

\author{
3 Daniel A. Nissley, Quyen V. Vu, Fabio Trovato, Nabeel Ahmed, Yang Jiang, Mai Suan Li,
} 4 and Edward P. O'Brien*

Cite This: https://dx.doi.org/10.1021/jacs.9b12264

Read Online

5 ABSTRACT: The ejection of nascent proteins out of the ribosome 6 exit tunnel after their covalent bond to transfer-RNA has been broken 7 and has not been experimentally studied due to challenges in sample 8 preparation. Here, we investigate this process using a combination of 9 multiscale modeling, ribosome profiling, and gene ontology analyses. 10 Simulating the ejection of a representative set of 122 E. coli proteins 11 we find a greater than 1000 -fold variation in ejection times. Nascent 12 proteins enriched in negatively charged residues near their C13 terminus eject the fastest, while nascent chains enriched in positively 14 charged residues tend to eject much more slowly. More work is 15 required to pull slowly ejecting proteins out of the exit tunnel than 16 quickly ejecting proteins, according to all-atom simulations. An

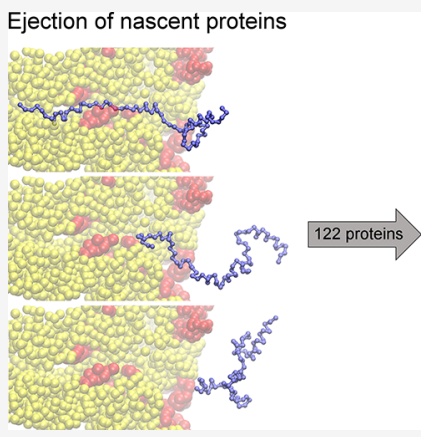

17 energetic decomposition reveals, for slowly ejecting proteins, that this is due to the strong attractive electrostatic interactions 18 between the nascent chain and the negatively charged ribosomal-RNA lining the exit tunnel, and for quickly ejecting proteins, it is 19 due to their repulsive electrostatic interactions with the exit tunnel. Ribosome profiling data from E. coli reveals that the presence of 20 slowly ejecting sequences correlates with ribosomes spending more time at stop codons, indicating that the ejection process might 21 delay ribosome recycling. Proteins that have the highest positive charge density at their C-terminus are overwhelmingly ribosomal 22 proteins, suggesting the possibility that this sequence feature may aid in the cotranslational assembly of ribosomes by delaying the 23 release of nascent ribosomal proteins into the cytosol. Thus, nascent chain ejection times from the ribosome can vary greatly 24 between proteins due to differential electrostatic interactions, can influence ribosome recycling, and could be particularly relevant to 25 the synthesis and cotranslational behavior of some proteins.

\section{6}

27 Translation is the process by which a protein is synthesized 28 from an mRNA and is carried out by the ribosome molecular 29 machine. The four phases of translation (initiation, elongation, 30 termination, and ribosome recycling) are areas of intense 31 research due to the essential role of protein synthesis in life. 32 Each phase is composed of multiple steps, many of which have 33 been characterized in terms of the structures adopted by the 34 molecules involved, the mechanisms of conformational and 35 chemical transitions, and the rates associated with these 36 transitions. ${ }^{1}$ Translation termination in E. coli, for example, 37 consists of some four steps: the binding of a release factor to a 38 stop codon in the A-site of the ribosome, the hydrolysis of the 39 covalent bond connecting the $\mathrm{C}$-terminus of the nascent chain 40 to the P-site tRNA, the ejection of the nascent protein out of 41 the exit tunnel, and the dissociation of the release factor from 42 the ribosome (Figure 1). While rates for release factor binding 43 and hydrolysis have been measured, ${ }^{2-6}$ the diffusion of the 44 nascent chain out of the exit tunnel has not been 45 experimentally characterized due to challenges with sample 46 preparation. Additionally, the presumably fast time scales of nascent chain ejection make experimental measurement a 47 challenge.

The ribosome exit tunnel is composed of both rRNA and 49 ribosomal protein and is therefore a chemically heterogeneous 50 environment through which nascent proteins pass into the 51 cytosol. At approximately $10 \mathrm{~nm}$ long and roughly $1.5 \mathrm{~nm}$ in 52 diameter, the interactions between the exit tunnel and nascent 53 chains can exhibit the full range of intermolecular forces, 54 including charge-charge, hydrogen bonding, and hydrophobic 55 interactions. Highly attractive forces can exist between some 56 regions of the exit tunnel and some nascent peptide 57 sequences. ${ }^{7}$ Indeed, peptide sequences known as stalling 58 sequences have evolved to take advantage of these interactions 59 and bind to the tunnel wall so tightly that they drastically slow 60

Received: December 2, 2019

Published: March 6, 2020 

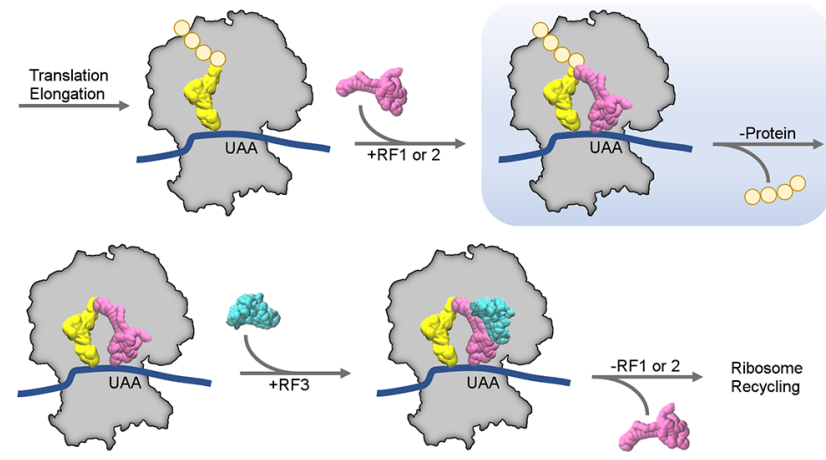

Figure 1. Translation termination in E. coli. Translation termination begins after translation elongation ends when a stop codon (e.g., UAA) enters the ribosome's A-site (ribosome shown as a gray outline). Release factor (RF) 1 or 2 (magenta) binds the stop codon in the A-site and catalyzes the hydrolysis of the peptidyl-tRNA bond between the nascent protein (orange spheres) and P-site tRNA (yellow). The nascent protein then diffuses out of the exit tunnel of the ribosome, which is around $10 \mathrm{~nm}$ in length. Following ejection, RF3 (cyan) catalyzes the release of RF1 or 2, allowing translation to proceed to the final phase, ribosome recycling. This study focuses on the second panel in this figure, highlighted in light blue. P-site tRNA and RF1 structures are generated from PDB ID 3OSK. The RF3 structure is generated from PDB ID 2H5E.

61 translation elongation, ${ }^{8,9}$ the biological benefit of which is to 62 regulate downstream protein synthesis. When stretches of 63 positively charged residues are present in the exit tunnel, they 64 slow protein elongation under both in vitro ${ }^{10}$ and in vivo 65 conditions. Since nascent protein elongation and nascent 66 protein ejection both involve the passage of nascent protein 67 segments through the exit tunnel, the interactions that can be 68 large and impactful during elongation also have the potential to 69 be important during ejection.

70 In this study, we use a combination of coarse-grained and 71 all-atom simulations, ribosome profiling data, and gene 72 ontology analysis to estimate the relative range of ejection 73 time scales that can occur across the cytosolic proteome of $E$. $74 \mathrm{coli}$, determine the intermolecular forces that give rise to the 75 extremes of ejection times, find experimental evidence that 76 slowly ejecting sequences can delay later stages of translation, 77 and identify nascent ribosomal proteins as some of the slowest 78 ejecting proteins from the ribosome.

\section{SIMULATION METHODS}

80 Single-Domain Protein Selection and Model Building. The 81 database from which the 122 proteins were selected contains 1014 82 cytosolic protein structures, 598 of which were single-domain proteins 83 and the rest multidomain proteins. ${ }^{11} \mathrm{~A}$ domain in this database is 84 classified as either $\alpha$ or $\beta$ if more than $70 \%$ of its residues identified by 85 STRIDE $^{12}$ to be in secondary structural elements were in $\alpha$-helixes or $86 \beta$-strands, respectively. Domains that simultaneously had $\alpha$-helical and $87 \beta$-strand content greater than $30 \%$ were classified as $\alpha / \beta .{ }^{11}$ Given the 88 sequence length distribution of these proteins, we determined that we 89 could feasibly simulate the synthesis and ejection of 122 proteins in 90 total. To maintain the ratio of single- to multidomain proteins in the 91 database, we selected 72 single-domain proteins and 50 multidomain 92 proteins. Of the 598 single-domain proteins in the database there are $93250 \alpha, 55 \beta$, and $293 \alpha / \beta$ domains; this ratio of structural classes was 94 reproduced in the subset of 72 single-domain proteins by randomly 95 selecting $30 \alpha, 7 \beta$, and $35 \alpha / \beta$ proteins (Tables S1 and S2). PDB files 96 for each single-domain protein were retrieved from the Protein Data 97 Bank, $^{13}$ and their corresponding mRNA sequences (NCBI assembly 98 eschColi_K12) were retrieved using the University of California Santa
Cruz microbe table browser (http://microbes.ucsc.edu/). Randomly 99 selected PDBs from the database were accepted only if the crystallized 100 sequence had no amino acid mutations in comparison to the amino 101 acid sequence which would result from the translation of the 102 eschColi_K12 mRNA. However, small sections of amino acids (12 or 103 less) or small numbers of heavy atoms (less than 10) that were not 104 resolved in the experimental structure were rebuilt on the basis of the 105 reference genome sequence and minimized in CHARMM. ${ }^{14} \quad 106$

Multidomain Protein Selection and Model Building. Fifty 107 multidomain proteins were selected randomly from the same 108 previously published database of E. coli globular proteins from 109 which single-domain proteins were selected. ${ }^{11}$ These multidomain 110 proteins are listed in Table S3. The amino acid sequence of each PDB 111 was aligned to the translated sequence of the corresponding gene in 112 NCBI assembly eschColi_K12, and missing residues and domains 113 were identified. Some PDBs contained large missing sections; to fill in 114 these regions with reasonable structures, PDBs representing the same 115 gene product were used to reconstruct missing sections after 116 structural alignment in VMD. ${ }^{15}$ PREDATOR $^{16}$ and IUPRED ${ }^{17} 117$ were used to predict whether those residues not resolved in any 118 other PDB were intrinsically unstructured, in which case they were 119 rebuilt and minimized in CHARMM rather than templated using 120 other structures. When homologous structures from E. coli were not 121 available, homologous structures from other organisms were used as a 122 template for the protein model, provided the sequence similarity was 123 greater than $30 \%$ and the backbone RMSD between the regions 124 common to the two structures was $\leq 2 \AA$.

Reconstructing missing domains or sections of the multidomain 126 proteins in this way resulted in models that still had, in some cases, 127 sections of missing atoms or mismatched amino acids relative to the 128 consensus mRNA sequence. All proteins were therefore subjected to a 129 rebuilding phase to add missing atoms and correct mutations or 130 sequence mismatches (Table S4). Because the reconstructed 131 segments were generated in an extended conformation, minimization 132 was performed in vacuo for 200 steps. This short minimization was 133 sufficient to resolve steric clashes. For proteins with short stretches of 134 missing residues (less than 10), this minimized configuration was 135 accepted as the final atomistic model. If a protein contained one or 136 more long stretches of missing residues (more than 10) or 137 disconnected domains, then the minimized protein structure was 138 subjected to additional dynamics at $310 \mathrm{~K}$. In this phase, the 139 reconstructed atoms within each templated domain were left free to 140 move, thereby allowing the structure to locally equilibrate. The 141 smallest domain in each protein was also left unrestrained in order to 142 allow it to reorient with respect to all other domains into a favorable 143 conformation. All other atoms were either held fixed or harmonically 144 restrained to the experimentally solved structure with a force constant 145 of $1 \mathrm{kcal} /\left(\mathrm{mol} \cdot \AA^{2}\right)$. All reconstructions, minimizations, and molecular 146 dynamics simulations were performed using CHARMM with the 147 par27 force field. ${ }^{14}$ The minimized structures were solvated in 148 TIP3P ${ }^{18}$ water and $150 \mathrm{mM} \mathrm{NaCl}$, gradually heated to $310 \mathrm{~K}$ for 100149 ps, and then equilibrated for $1.5 \mathrm{~ns}$ at the same temperature. 150 Production runs had different durations, spanning from 20 to $50 \mathrm{~ns} .151$ Langevin dynamics with a friction coefficient of $1.0 \mathrm{ps}^{-1}$ and a time 152 step of $1.5 \mathrm{fs}$ were used. For each protein, the conformation with the 153 lowest potential energy was selected as the final atomistic model. We 154 emphasize that the purpose of the molecular dynamics simulations 155 was not to thoroughly explore the conformational space of the 156 multidomain proteins but rather to provide reasonable atomistic 157 conformations for building coarse-grained models.

Domains in the multidomain proteins were initially defined 159 according to $\mathrm{CATH},{ }^{19}$ which is also used in the original database. ${ }^{11} 160$ Domain residue numberings were shifted to match the translated 161 sequence and modeling of the missing atoms performed (Table S4). 162 The final domain definitions reported in Table S3 include residues 163 and domains that were modeled as described in Table S4.

Coarse-Grained Force Field and Model Construction. The 165 potential energy for a given configuration of the $\mathrm{C}_{\alpha}$ coarse-grained 166 model is calculated using the equation 


$$
\begin{aligned}
& E=\sum_{i} k_{\mathrm{b}}\left(r_{i}-r_{0}\right)^{2}+\sum_{i} \sum_{j=1}^{4} k_{\varphi, i j}\left(1+\cos \left[j \varphi_{i}-\delta_{i j}\right]\right) \\
& +\sum_{i}-\frac{1}{\gamma} \ln \left\{\exp \left[-\gamma\left(k_{\alpha}\left(\theta_{i}-\theta_{\alpha}\right)^{2}+\varepsilon_{\alpha}\right)\right]+\exp \left[-\gamma k_{\beta}\left(\theta_{i}-\theta_{\beta}\right)^{2}\right]\right\} \\
& +\sum_{i j} \frac{q_{i} q_{j} \mathrm{e}^{2}}{4 \pi \varepsilon_{0} \varepsilon_{\mathrm{r}} r_{i j}} \exp \left[-\frac{r_{i j}}{l_{\mathrm{D}}}\right]+\sum_{i j \in\{\mathrm{NC}\}} \epsilon_{i j}^{\mathrm{NC}}\left[13\left(\frac{\sigma_{i j}}{r_{i j}}\right)^{12}-18\left(\frac{\sigma_{i j}}{r_{i j}}\right)^{10}+4\left(\frac{\sigma_{i j}}{r_{i j}}\right)^{6}\right] \\
& +\sum_{i j \notin\{\mathrm{NC}\}} \epsilon_{i j}^{\mathrm{NN}}\left[13\left(\frac{\sigma_{i j}}{r_{i j}}\right)^{12}-18\left(\frac{\sigma_{i j}}{r_{i j}}\right)^{10}+4\left(\frac{\sigma_{i j}}{r_{i j}}\right)^{6}\right]
\end{aligned}
$$

168 The terms in this equation represent, from left to right, summations 169 over the contributions from $\mathrm{C}_{\alpha}-\mathrm{C}_{\alpha}$ bonds, dihedral angles, bond 170 angles, electrostatic interactions, Lennard-Jones-like native interac171 tions, and repulsive non-native interactions to the total potential 172 energy. The bond, dihedral, and angle terms have been described in 173 detail elsewhere. ${ }^{20,21}$ Electrostatics are treated using Debye-Hückel 174 theory with a Debye length, $l_{\mathrm{D}}$, of $10 \AA$ and a dielectric of 78.5 ; lysine 175 and arginine $\mathrm{C}_{\alpha}$ sites are assigned $q=+\mathrm{e}$, glutamic acid and aspartic 176 acid are assigned $q=-\mathrm{e}$, and all other interaction sites are 177 uncharged. ${ }^{22}$ The contribution from native interactions is computed 178 using the $12-10-6$ potential of Karanicolas and Brooks. ${ }^{20}$ The value 179 of $\epsilon_{i j}^{\mathrm{NC}}$, which sets the depth of the energy minimum for a native 180 contact, is calculated as $\epsilon_{i j}^{\mathrm{NC}}=n_{i j} \epsilon_{\mathrm{HB}}+\eta \epsilon_{i j}$. Here, $\epsilon_{\mathrm{HB}}$, and $\epsilon_{i j}$ represent 181 energy contributions arising from hydrogen bonding and van der 182 Waals contacts between residues $i$ and $j$ identified from the all-atom 183 structure of the protein, respectively. $n_{i j}$ is the number of hydrogen 184 bonds formed between residues $i$ and $j$ and $\epsilon_{\mathrm{HB}}=0.75 \mathrm{kcal} / \mathrm{mol}$. The 185 value of $\epsilon_{i j}$ is set on the basis of the Betancourt-Thirumalai pairwise 186 potential. ${ }^{23}$ The scaling factor $\eta$ is determined for each of our 122 187 proteins (Tables S2 and S3) based on a previously published training $188 \mathrm{set}^{24}$ to reproduce realistic protein stabilities for different structural 189 classes (Table S5, Table S6, and Supplementary Methods). A single 190 value of $\eta$ is applied to all native contacts for a given single-domain 191 protein and for each individual domain and interface in multidomain 192 proteins. Collision diameters, $\sigma_{i j}$, between $\mathrm{C}_{\alpha}$ interactions sites 193 involved in native contacts are set equal to the distance between 194 the $\mathrm{C}_{\alpha}$ of the corresponding residues in the crystal structure divided 195 by $2^{1 / 6}$. For non-native interactions, $\epsilon_{i j}^{\mathrm{NN}}$ is set to $0.000132 \mathrm{kcal} / \mathrm{mol}$ 196 and $\sigma_{i j}$ is computed as previously reported. ${ }^{20}$

197 Simulations of Nascent Protein Synthesis and Ejection. The 198 coarse-grained model of each protein in the single- and multidomain 199 protein data sets was synthesized starting from a single residue (see 200 the two exceptions below) using a modified version of a previously 201 published protocol on a coarse-grained representation of the 50S E. 202 coli ribosome (details of the ribosome model can be found in 203 Supplementary Methods). ${ }^{25}$ The dwell time at a particular nascent 204 chain length was randomly selected from an exponential distribution 205 with a mean equal to the average decoding time of the codon in the 206 A-site. Average decoding times are taken from the Fluitt-Viljoen 207 model $^{26}$ and scaled to reproduce an overall average of $12.6 \mathrm{~ns}$ 208 (840 000 integration time steps of 0.015 ps duration; see Table S7) 209 based on a previously published training set. ${ }^{24}$ A planar restraint in 210 the $y z$ plane through the point $(58,0,0) \AA$ is used to prevent the 211 nascent chain from contacting the underside of the ribosome cutout. 212 Fifty trajectories were run for each of the 122 proteins in the data set. 213 After synthesis was completed for a given trajectory, the harmonic 214 restraint on the C-terminal bead to model the covalent bond between 215 the nascent protein and the P-site tRNA was removed. Simulations of 216 termination were run until the C-terminal residue of each trajectory 217 reached an $x$ coordinate of $100 \AA$ or greater, indicating that the 218 protein exited the tunnel. Ejection times are calculated as the time 219 between when the $\mathrm{C}$-terminal harmonic restraint is removed and 220 when the C-terminal residue reaches an $x$ coordinate of $\geq 100 \AA$. Two 221 proteins (PDB IDs $2 \mathrm{KFW}$ and $3 \mathrm{GN} 5$ ) became stalled in the exit 222 tunnel when synthesis was begun from a single residue; synthesis for 223 these two proteins was therefore initiated from a nascent chain length 224 of 50 residues. One protein (PDB ID 4DCM) did not eject from the exit tunnel in 27 of 50 trajectories during 25 days of CPU time when 225 its wild-type C-terminal charges were used; the ejection time for this 226 protein is therefore reported as a lower bound. Mean ejection times 227 for all 122 proteins are listed in Table S8.

All-Atom Steered Molecular Dynamics Simulations. The 50S 229 subunit of the E. coli ribosome (PDB ID 3R8T) was aligned with the 230 long axis of the exit tunnel, defined to be between atom N6 of 231 nucleotide $\mathrm{A} 2602$ and the $\mathrm{C}_{\beta}$ atom of Ala50 in ribosomal protein L24, 232 along the $x$ axis of the simulation coordinate system. The ribosome 233 was then cropped to form a rectangular box around the exit tunnel 234 with dimensions of $13.10590 \times 8.44869 \times 8.18680 \mathrm{~nm}^{3}$. Coarse- 235 grained structures of the C-terminal 30 aa of nascent proteins from 236 the final time step of synthesis simulations were backmapped to 237 atomistic resolution for use as starting structures. The first step in 238 backmapping is the insertion of coarse-grained sites representing 239 amino acid side chains near their corresponding $\mathrm{C}_{\alpha}$ beads followed by 240 energy minimization in the $\mathrm{C}_{\alpha}$ side-chain model force field ${ }^{27}$ with all 241 $\mathrm{C}_{\alpha}$ positions restrained. Backbone and side-chain all-atom structures 242 were then rebuilt using Prodart2 ${ }^{28}$ and Pulchra, ${ }^{29}$ respectively, on the 243 minimized $\mathrm{C}_{\alpha}$ side-chain model. The final backmapped structure was 244 obtained after energy minimization within the generalized Born (GB) 245 implicit water environment. ${ }^{30}$ The $\mathrm{N}$-terminus of the segment was 246 capped by the N-terminal acetyl capping group (ACE) and the 247 atomistic protein structure inserted into the atomistic exit tunnel 248 structure.

A simulation box was constructed with a minimum of $1 \mathrm{~nm} 250$ between the edge of the cropped ribosome and the periodic boundary 251 wall in all dimensions and then extended $15 \mathrm{~nm}$ in the positive $x 252$ dimension to accommodate the nascent protein when fully extracted 253 from the exit tunnel at the end of the steered molecular dynamics 254 simulation. The system was neutralized with $\mathrm{Na}^{+}$before adding $5 \mathrm{mM} 255$ $\mathrm{MgCl}_{2}$ and $100 \mathrm{mM} \mathrm{NaCl}$. Next, the system was minimized in the gas 256 phase with the steepest-descent algorithm. Harmonic restraints on all 257 $\mathrm{C}_{\alpha}$ atoms of the nascent peptide and all heavy atoms of the ribosome 258 with a force constant of $1000 \mathrm{~kJ} /\left(\mathrm{mol} \cdot \mathrm{nm}^{2}\right)$ were employed to 259 prevent the nascent protein from moving during minimization. The 260 system was then equilibrated in the gas phase for 300 ps to allow ions 261 to rapidly find binding sites on the ribosome, with harmonic restraints 262 again applied to $\mathrm{C}_{\alpha}$ atoms of the nascent chain and all heavy atoms of 263 the ribosome.

The cropped ribosome and nascent protein were then solvated and 265 equilibrated. First, 1 ns of dynamics was carried out in the NVT 266 ensemble, followed by $1 \mathrm{~ns}$ of dynamics in the NPT ensemble, with 267 the temperature and pressure held at $310 \mathrm{~K}$ and $1 \mathrm{~atm}$, respectively. 268 To allow the nascent protein and the ribosome exit tunnel to reach 269 equilibrium in the all-atom model, we performed a second NPT 270 simulation for $10 \mathrm{~ns}$ with harmonic restraints applied to $\mathrm{P}$ and $\mathrm{C}_{\alpha} 271$ atoms of the ribosome that were more than $28 \AA$ from the $x$ axis and 272 all $\mathrm{C}_{\alpha}$ atoms of the nascent protein. The center of mass of the N- 273 terminal ACE residue was then pulled from the exit tunnel with a 274 cantilever speed of $0.25,1$, or $5 \mathrm{~nm} / \mathrm{ns}$ and a spring constant of 600275 $\mathrm{kJ} /\left(\mathrm{mol} \cdot \mathrm{nm}^{2}\right)$. All simulations were carried out with GROMACS 276 $2018^{31}$ using the AMBER99SB ${ }^{32}$ force field and the TIP3P ${ }^{18}$ water 277 model. The particle mesh Ewald method ${ }^{33}$ was used to calculate the 278 long-range electrostatic interactions beyond $1.2 \mathrm{~nm}$. Lennard-Jones 279 interactions were calculated within a distance of $1.2 \mathrm{~nm}$. The Nose- 280 Hoover thermostat ${ }^{34,35}$ and Parrinello-Rahman barostat ${ }^{36}$ were 281 employed to maintain the temperature and pressure at $310 \mathrm{~K}$ and 1282 atm, respectively. The LINCS algorithm ${ }^{37}$ was used to constrain all 283 bonds, and the integration time step was set to $2 \mathrm{fs}$.

Simulations were carried out using this protocol for 5 quickly 285 ejecting proteins (PDB IDs 1FM0, 1Q5X, 1T8K, 2KFW, and 3BMB) 286 and 5 slowly ejecting proteins (PDB IDs 1AH9, 1JW2, 2JO6, 2PTH, 287 and 3IV5) using 21 different initial configurations from the coarse- 288 grained synthesis simulations for each different protein.

\section{RESULTS AND DISCUSSION}

To estimate the range of nascent chain ejection times across 291 different proteins, we simulated the synthesis and ejection of 292 
a

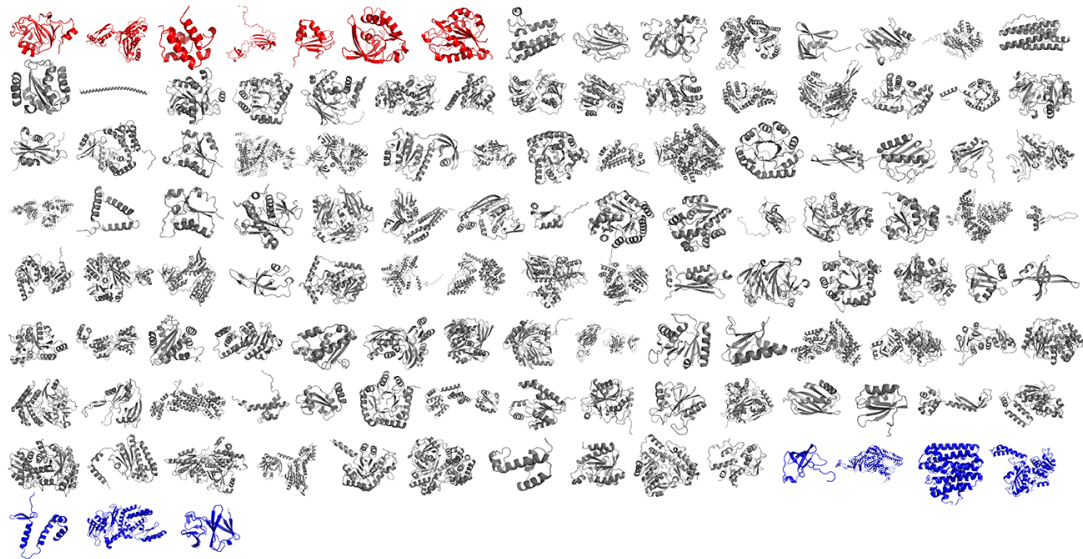

b

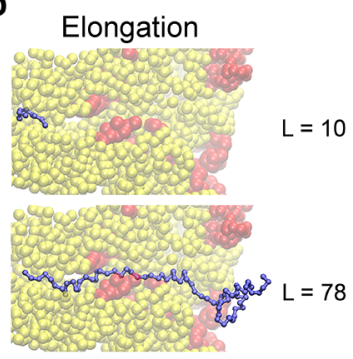

C

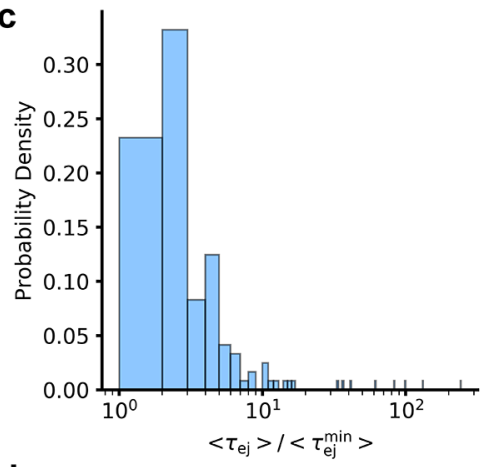

d

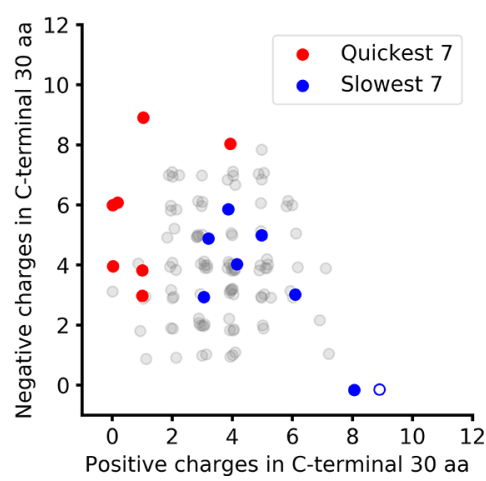

Figure 2. The 242-fold variation in ejection times is related to the presence of charged residues in the C-termini of proteins. (a) The set of $122 \mathrm{E}$. coli proteins that were simulated is shown from top left to bottom right by increasing ejection time. The top 5\% fastest and slowest ejecting proteins are colored red and blue, respectively, while the middle $90 \%$ are colored gray. (b) Coarse-grained simulations begin with the elongation phase, during which the protein (blue) is synthesized on the ribosome (rRNA and protein colored yellow and red, respectively). Once the full-length protein is synthesized, ejection occurs. Ejection is complete once the C-terminal residue is $100 \AA$ from the peptidyl transferase center of the ribosome. (c) Distribution of mean ejection times, $\left\langle\tau_{\mathrm{ej}}\right\rangle$, for 121 of the 122 proteins shown in (a) (excluding 4DCM), normalized by the factor $\left\langle\tau_{\mathrm{ej}}^{\mathrm{min}}\right\rangle$ which is the smallest $\left\langle\tau_{\mathrm{ej}}\right\rangle$ found in the set of proteins. (d) Number of positive and negative charges in each protein's C-terminal 30 residues. The fastest and slowest ejectors (bottom and top 5\% of the distribution in panel c) are colored red and blue, respectively, and exist as separate, nonoverlapping populations along these metrics. Values from other proteins are displayed in transparent gray. Random noise (jitter) has been added to minimize overlapping points. As discussed in the main text, the single unfilled blue data point at 9 positive charges and zero negative charges is for PDB ID 4DCM, for which an exact ejection time could not be calculated because not all of its trajectories were released from the ribosome in the simulation. Therefore, this protein was excluded from the distribution in panel $\mathrm{c}$.

293122 full-length E. coli proteins using a coarse-grained 294 representation of the ribosome nascent chain complex (Figure $2952 \mathrm{a}, \mathrm{b}) .^{25,27,38,39}$ This set of 122 proteins is representative of the 296 globular E. coli cytosolic proteome as a whole because it 297 reproduces the proteome-wide protein size and structural class 298 distributions (Figure S1, Table S1, and Simulation Methods). 299 Fifty statistically independent synthesis and ejection trajecto300 ries were run for each protein. We find that the mean protein 301 ejection time, defined as the average time it takes for the C302 terminal nascent chain residue to reach the end of the exit 303 tunnel after the bond between the protein and P-site tRNA is 304 broken, varies 242-fold across 121 of these proteins (Figure $3052 \mathrm{c}$ ). We observed that the proteins at the extremes of this 306 ejection time distribution, that is, those proteins in the top and 307 bottom 5\%, have markedly different electrostatic characteristics 308 in their C-termini (Figure $2 \mathrm{~d}$ ), the last 30 residues of which are 309 in the exit tunnel. Quickly ejecting proteins tend to have 310 abundant negatively charged residues and few positively 311 charged residues in their C-terminal 30 residues (red dots in 312 Figure 2d). In contrast, slowly ejecting proteins tend to have 313 fewer negatively charged residues and more positively charged 314 residues (blue dots in Figure 2d). We note the exceptional case 315 of the protein with PDB ID 4DCM (unfilled blue point in 316 Figure $2 \mathrm{~d}$ ), which is the $122 \mathrm{nd}$ protein in our set. (Note that 317 complete protein names are provided in Tables S2 and S3.) 318 While complete ejection occurred for all other proteins, only
23 out of the 50 simulation trajectories of 4DCM were fully 319 ejected from the exit tunnel. Under a conservative estimate, 320 this protein's average ejection time is 7031-fold slower than the 321 fastest ejecting protein in our data set. Consistent with 322 electrostatics being important, 4DCM also has the greatest 323 positive charge density in our set of proteins. These results 324 indicate that there is a 3-order-of-magnitude spread in ejection 325 times across E. coli cytosolic proteins and suggest that the very 326 fast ejectors are fast because they are electrostatically repelled 327 by the exit tunnel, which is lined with negatively charged 328 rRNA, while the very slow ejectors are slow because they are 329 electrostatically attracted to the exit tunnel wall.

To test this electrostatic hypothesis within our coarse- 331 grained model, we set to zero all negative or positive charges of 332 amino acids in the C-terminal 30 residues of the quickly or 333 slowly ejecting proteins, respectively. All other interactions and 334 charges involving the ribosome and nascent chain remained 335 the same. Rerunning the ejection simulations for these 336 sequences, we find that removing negative charges from the 337 set of quick ejectors slowed the ejection process by 5-98\% 338 (average 44\%) and removing positive charges from the set of 339 slowly ejecting proteins sped up the ejection process by $48-340$ $99 \%$ (average $82 \%$, 4DCM results excluded) (Table 1). These $341 \mathrm{t} 1$ results are consistent with the hypothesis that electrostatic 342 interactions are a causal factor in influencing extremely fast or 343 extremely slow ejection times out of the ribosome tunnel. 344 
Table 1. Ejection Times upon Neutralization of C-Terminal Positive or Negative Residues

\begin{tabular}{|c|c|c|c|}
\hline \multicolumn{4}{|c|}{ quickly ejecting peptides } \\
\hline PDB ID & wild type (ns) & no $(-)$ charges $(\mathrm{ns})^{a}$ & $\%$ change \\
\hline 1Q5X & 0.31 & 0.47 & 51.9 \\
\hline $3 \mathrm{M} 7 \mathrm{M}$ & 0.31 & 0.41 & 30.8 \\
\hline $1 \mathrm{~T} 8 \mathrm{~K}$ & 0.32 & 0.42 & 31.9 \\
\hline $2 \mathrm{KFW}$ & 0.32 & 0.41 & 27.3 \\
\hline 1FM0 & 0.36 & 0.38 & 4.55 \\
\hline 3BMB & 0.37 & 0.57 & 51.8 \\
\hline 1AG9 & 0.39 & 0.76 & 95.8 \\
\hline $2 \mathrm{HGK}$ & 0.40 & 0.65 & 62.3 \\
\hline $1 \mathrm{FJJ}$ & 0.41 & 0.44 & 8.06 \\
\hline $2 \mathrm{HO} 9$ & 0.41 & 0.60 & 43.8 \\
\hline 1SVT & 0.42 & 0.50 & 19.4 \\
\hline 1SG5 & 0.45 & 0.88 & 98.0 \\
\hline \multicolumn{4}{|c|}{ slowly ejecting peptides } \\
\hline PDB ID & wild type (ns) & no $(+)$ charges $(\mathrm{ns})^{a}$ & $\%$ change \\
\hline 4IM7 & 3.92 & 0.69 & -82.3 \\
\hline 1JW2 & 4.39 & 1.94 & -55.9 \\
\hline $2 \mathrm{PTH}$ & 4.75 & 0.86 & -81.9 \\
\hline $1 \mathrm{D} 2 \mathrm{~F}$ & 5.02 & 0.64 & -87.2 \\
\hline $3 \mathrm{OFO}$ & 10.22 & 5.28 & -48.3 \\
\hline $1 \mathrm{AH} 9$ & 11.28 & 0.59 & -94.8 \\
\hline 1NG9 & 12.66 & 2.31 & -81.8 \\
\hline 1RQJ & 18.80 & 0.84 & -95.5 \\
\hline 1T4B & 25.66 & 2.58 & -90.0 \\
\hline 3IV5 & 30.47 & 0.45 & -98.5 \\
\hline 1U0B & 40.75 & 1.04 & -97.5 \\
\hline 2JO6 & 74.73 & 23.31 & -68.8 \\
\hline $4 \mathrm{DCM}$ & $>2170$ & 0.54 & -100.0 \\
\hline
\end{tabular}

${ }^{a}$ Columns labeled "no ( -$)$ charges" and "no $(+)$ charges" are ejection times from simulations in which negative or positive charges in $\mathrm{C}$ terminal 30 aa, respectively, are made electrically neutral.

345 While coarse-grained models can simulate larger systems for 346 longer times in comparison to all-atom simulations, they leave 347 out atomic details that have the potential to influence these 348 results. It is currently not possible to simulate the complete 349 ejection process of nascent chains from ribosomes using 350 unrestrained all-atom molecular dynamics simulations. There351 fore, to qualitatively test the robustness of the conclusions 352 from our coarse-grained model, we carried out nonequilibrium 353 all-atom steered molecular dynamics simulations in which the 354 nascent protein is pulled from the ribosome exit tunnel using 355 an external pulling force applied to the N-terminus of the 356 protein (Figure $3 \mathrm{a}$ ). If the coarse-grained model results are 357 correct, then we predict that it will be harder to pull (as 358 measured by the nonequilibrium work) the slowly ejecting 359 chains out of the exit tunnel as compared to the quickly 360 ejecting chains due to differential electrostatic interactions with 361 the ribosome exit tunnel. Twenty-one independent trajectories 362 were run for each of five quickly ejecting and five slowly 363 ejecting proteins drawn from the bottom and top $10 \%$ of the 364 distribution of ejection times, respectively. A cantilever speed 365 of $0.25 \mathrm{~nm} / \mathrm{ns}$ was used for all simulations. In these all-atom 366 simulations, we find that the slowly ejecting nascent proteins 367 require $28 \%$ (95\% CI [19\%, 36\%] computed from boot368 strapping; $p<1 \times 10^{-8}$ computed from the permutation test) 369 more work on average to be extracted from the exit tunnel than 370 quickly ejecting nascent proteins (Figure 3b,c). Decomposing a

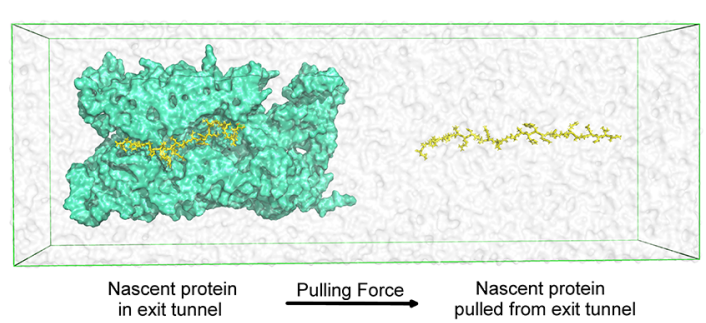

b

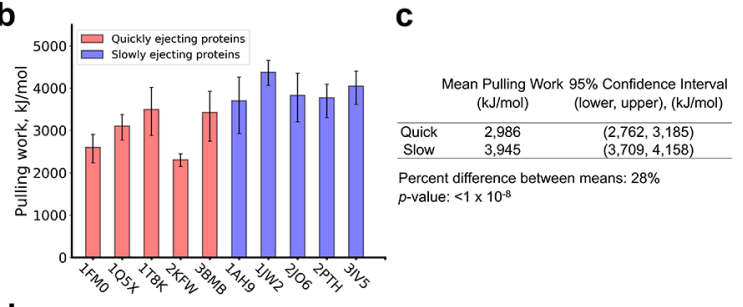

d

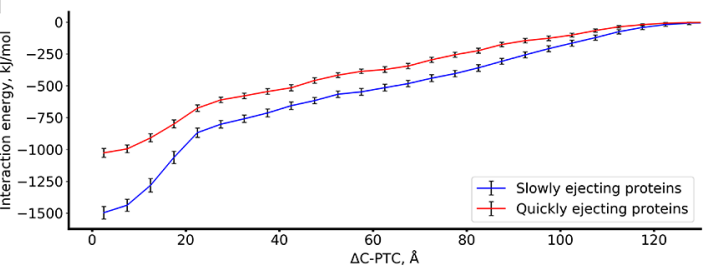

e
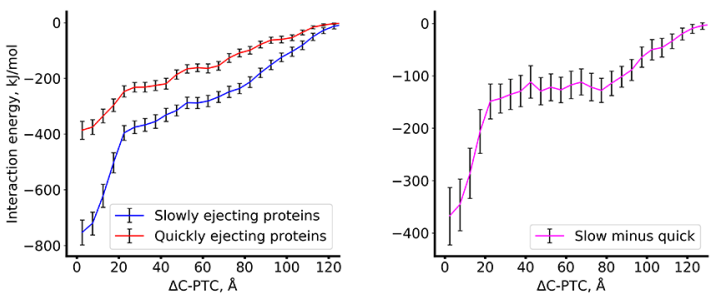

f
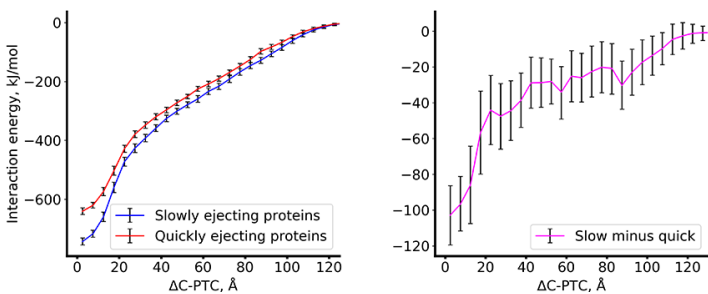

Figure 3. Slowly ejecting proteins are more electrostatically attracted to the ribosome exit tunnel. (a) Initial (left) and final (right) conformations from all-atom, steered molecular dynamics simulations of the extraction of a nascent protein (yellow) from the ribosome (cyan). (b) Mean pulling work required to extract 10 different nascent proteins from the ribosome exit tunnel from 21 statistically independent simulations per protein. Error bars are 95\% confidence intervals calculated by bootstrapping. (c) Results from the statistical comparison between the overall means of the slowly and quickly ejecting sets. Confidence intervals are calculated as in (b). The $p$ value is estimated using a permutation test. (d) Total interaction energy between the ribosome and nascent protein as a function $\Delta \mathrm{C}-\mathrm{PTC}$, the distance between the $\mathrm{C}_{\alpha}$ atom of the $\mathrm{C}$-terminal residue of the nascent protein and the N6 atom of nucleotide A2602 in the peptidyl transferase center of the ribosome. (e) Electrostatic contribution to the total interaction energy (left) and the difference between the slowly and quickly ejecting data set mean electrostatic interaction energies (right). (f) The same as in (d) but for the van der Waals interaction energy. These results were obtained using a cantilever speed of $0.25 \mathrm{~nm} / \mathrm{ns}$.

the intermolecular interactions in these simulations, we find 371 that slowly ejecting nascent proteins have stronger interactions 372 
373 with the ribosome tunnel wall than quickly ejecting proteins 374 (Figure $3 \mathrm{~d}-\mathrm{f}$ ), with the majority of this energy difference due 375 to electrostatic rather than van der Waals interactions (Figure $3763 \mathrm{e}, \mathrm{f})$. Qualitatively equivalent results are obtained when the 377 cantilever speed is increased 4 - or 20 -fold to 1 or $5 \mathrm{~nm} / \mathrm{ns}$, 378 respectively (Figures S2 and S3). Thus, the all-atom results and 379 coarse-grained results are consistent, lending further support to 380 the hypothesis that electrostatic interactions between the 381 nascent chain and ribosome govern the extremes of nascent 382 chain ejection times.

383 An important biological question is whether there are any 384 downstream consequences of this broad range of ejection 385 times. We hypothesized that the slowest ejecting sequences 386 might delay the onset of the next and final step of translation 387 (Figure 1), ribosome recycling, during which molecular factors 388 interact with the ribosome to aid the dissociation of the small 389 and large ribosomal subunits. This hypothesis predicts that 390 ribosomes will dwell for longer at stop codons when a slowly 391 ejecting sequence is present compared to when a quickly 392 ejecting sequence is present. To test this hypothesis, we 393 analyzed ribosome profiling data from E. coli ${ }^{40}$ of those 394 cytosolic proteins that have the highest charge density at their 395 C-terminus. Ribosome profiling is an experimental technique 396 that measures a signal, called the "reads", that is proportional 397 to the number of ribosomes sitting at a particular codon 398 position on the various cellular copies of an mRNA 399 transcript. $^{41}$ As such, the greater the normalized ribosome 400 density at a codon, the longer the ribosome spent at that codon 401 position. The normalized ribosome density at a codon position 402 is the number of reads at that codon divided by the average 403 number of reads per codon arising from the coding sequence 404 of the transcript. Therefore, our hypothesis predicts that there 405 will be greater ribosome density at the stop codon for proteins 406 that have the highest number of positive charges in their C407 terminus compared to those that have high negative charge 408 density. Therefore, we restricted our analysis to high-coverage 409 transcripts (Supplementary Methods) encoding proteins with 410 either $\geq 8$ positive and $\leq 2$ negative residues in their $30 \mathrm{C}$ 411 terminal residues, which we predict to be slowly ejecting 412 proteins ( $n=22$ proteins), or with $\geq 8$ negative residues and $413 \leq 2$ positively charged residues $(n=22$ proteins $)$ in their $30 \mathrm{C}$ 414 terminal residues, which we predict to be quickly ejecting 415 proteins. We could not include all of the fastest and slowest 416 ejecting proteins from our simulations in this analysis because 417 the read coverage of their transcripts was very sparse in the 418 ribosome profiling data, meaning that their signal-to-noise ratio 419 is too low to be useful. However, two proteins (PDB IDs 1T8K 420 and 3IV5) for which we simulated ejection times did have 421 sufficient read coverage and are included in this analysis. We 422 observe that the putative slow ejectors have on average 3.3-fold 423 higher ribosome density at the stop codon compared to the 424 fast ejectors (Figure 4a,b; median ribosome densities across 425 fast and slow ejector sets are, respectively, 0.248 and 0.812 ; the 426 difference between medians is significant based on the Mann427 Whitney U Test, $p=0.011$ ). These results are consistent with 428 the hypothesis that the slowest ejecting nascent chains tend to 429 delay ribosome recycling.

430 To further explore the potential biological ramifications of 431 very fast or slow ejection times, we carried out a gene ontology 432 analysis to determine whether putative slowly and quickly 433 ejecting proteins are more likely than random chance to be 434 associated with particular cellular or biochemical processes 435 (Supplementary Methods). The putative quickly ejecting
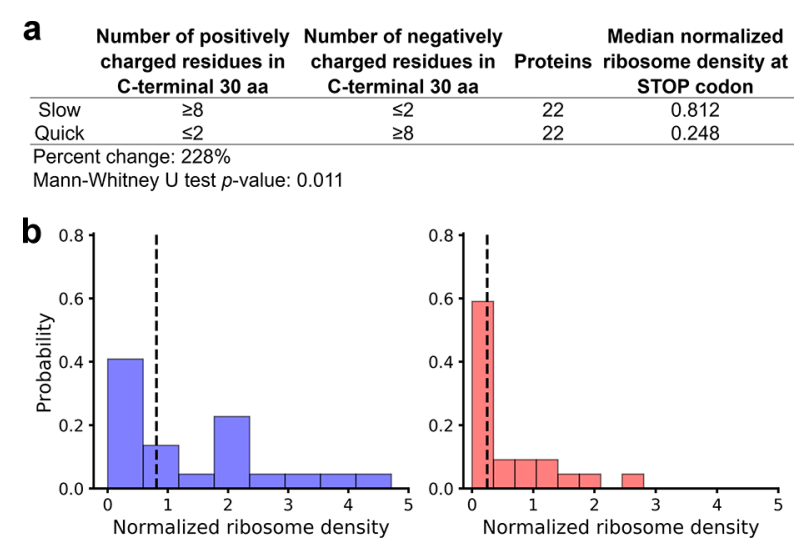

Figure 4. Presence of slowly ejecting proteins in ribosome-nascent chain complexes correlated with longer ribosome dwell times at the stop codon. (a) Proteins with $\geq 8$ positive and $\leq 2$ negative residues in their C-terminus as well as proteins with $\geq 8$ negative and $\leq 2$ positive residues in their $\mathrm{C}$-terminus were selected from the $E$. coli ribosome profiling data from ref 40 . A total of 22 proteins fit into each category. The median normalized ribosome density is higher for proteins enriched in positive charge at the C-terminus ( $p$ value 0.011 , MannWhitney U test). (b) Histograms of normalized ribosome density at the stop codon for the subsets of proteins enriched in positive (left, blue histogram) or negative (right, red histogram) amino acids.

proteins exhibit no significant relationship to any particular 436 biological processes. However, 14 of the 22 potentially slowly 437 ejecting proteins are associated with translation, and 13 are 438 ribosomal subunit proteins. Two hypotheses can explain this 439 observation. First, slow ejection increases the time a nascent 440 protein is available for cotranslational assembly, ${ }^{42-44}$ suggest- 441 ing that these highly positively charged C-terminal segments 442 might have evolved to aid in the efficient cotranslational 443 assembly of ribosomes in the E. coli cytosol. Second, ribosomal 444 proteins may have evolved positively charged segments solely 445 to aid in their interactions with rRNA in the context of a fully 446 assembled ribosomal subunit, with their slow ejection times 447 being a biologically irrelevant consequence of this fact. Indeed, 448 each of the 13 ribosomal proteins identified by this analysis is 449 in contact with rRNA based on the analysis of a crystal 450 structure of the E. coli ribosome in the nonrotated 451 conformation (PDB ID 4V9D). It will be an interesting area 452 of future research to test these distinct hypotheses.

\section{CONCLUSIONS}

453

Our results indicate that nascent protein ejection times are 455 very broad, that the extremes are primarily driven by 456 interactions of the high charge density of either positive or 457 negative residues near the nascent protein's C-terminus with 458 the negatively charged ribosome exit tunnel, and that very 459 slowly ejecting chains can delay ribosome recycling. The fact 460 that ribosomal proteins have some of the most highly positively 461 charged C-termini across the E. coli proteome suggests the 462 intriguing possibility that their charge density did not evolve 463 just to strengthen their binding affinity for rRNA but could 464 also be beneficial by making them slow ejectors, thereby 465 affording more time for potential cotranslational assembly 466 processes to occur. While we have demonstrated that 467 electrostatics are essential for extreme ejection times by 468 running simulations without the charges present in the nascent 469 chain C-termini, other factors must also play a role in 470 determining ejection times. As can be seen in Figure 2d, some 471 
472 proteins with typical ejection times (gray dots) have a similar 473 number of positive charges in the $\mathrm{C}$-terminus as proteins with 474 very slow ejection times. We speculate that other factors that 475 influence the ejection time could include the backbone 476 structural propensity and the size of the amino acids in the 477 protein sequence. Helical backbone preferences and large 478 amino acids are more likely to sterically clash with the walls of 479 the exit tunnel, while extended strand backbone preferences 480 and small amino acids might make diffusion out of the tunnel 481 sterically easier. This study is the first to our knowledge to 482 provide evidence that the seemingly mundane act of diffusion 483 of nascent proteins out of the exit tunnel can vary greatly 484 between proteins, have downstream cellular consequences, and 485 might be particularly biologically relevant to the synthesis of 486 ribosomal proteins.

\section{ASSOCIATED CONTENT}

\section{SI Supporting Information}

489 The Supporting Information is available free of charge at 490 https://pubs.acs.org/doi/10.1021/jacs.9b12264.

491 Full details of coarse-grained model parametrization and 492 simulations, additional all-atom steered molecular 493 dynamics results with different cantilever speeds, and $494 \quad$ GO pathway analysis details (PDF)

$495 \quad$ Fast ejection (AVI)

496 Intermediate ejection (AVI)

$497 \quad$ Slow ejection (AVI)

498 AUTHOR INFORMATION

499 Corresponding Author

500 Edward P. O'Brien - Department of Chemistry, Bioinformatics 501 and Genomics Graduate Program, The Huck Institutes of the

502 Life Sciences, and Institute for Computational and Data

503 Sciences, Pennsylvania State University, University Park,

504 Pennsylvania 16802, United States; (1) orcid.org/0000-0001-

50599809-3273; Email: epo2@psu.edu

\section{Authors}

507 Daniel A. Nissley - Department of Chemistry, Pennsylvania

508 State University, University Park, Pennsylvania 16802, United $509 \quad$ States

510 Quyen V. Vu - Institute of Physics, Polish Academy of Sciences, 511 02-668 Warsaw, Poland; (1) orcid.org/0000-0002-9863-0486

512 Fabio Trovato - Department of Chemistry, Pennsylvania State 513 University, University Park, Pennsylvania 16802, United States

514 Nabeel Ahmed - Department of Chemistry and Bioinformatics 515 and Genomics Graduate Program, The Huck Institutes of the 516 Life Sciences, Pennsylvania State University, University Park, $517 \quad$ Pennsylvania 16802, United States

518 Yang Jiang - Department of Chemistry, Pennsylvania State

519 University, University Park, Pennsylvania 16802, United

520 States; (i) orcid.org/0000-0003-1100-9177

521 Mai Suan Li - Institute of Physics, Polish Academy of Sciences, 522 02-668 Warsaw, Poland; Institute for Computational Sciences 523 and Technology, Ho Chi Minh City, Vietnam; ocid.org/ $524 \quad$ 0000-0001-7021-7916

525 Complete contact information is available at:

526 https://pubs.acs.org/10.1021/jacs.9b12264

527 Notes

528 The authors declare no competing financial interest.

\section{ACKNOWLEDGMENTS}

529

E.P.O. acknowledges support from the National Science 530 Foundation (MCB-1553291) for the simulation component 531 and (ABI-1759860) for the bioinformatics component of this 532 study, as well as the National Institutes of Health (R35- 533 GM124818). Portions of numerical computations and data 534 analysis in this work have been carried out on the CyberLAMP 535 cluster, which is supported by NSF-MRI-1626251 and 536 operated by the Institute for CyberScience at The 537 Pennsylvania State University. This work used the Extreme 538 Science and Engineering Discovery Environment (XSEDE), 539 which is supported by National Science Foundation grant 540 number ACI-1548562. M.S.L. acknowledges that this work was 541 supported by Narodowe Centrum Nauki (grant no. 2015/19/ 542 B/ST4/02721), PLGrid Infrastructure in Poland, and the 543 Department of Science and Technology, Ho Chi Minh City, 544 Vietnam.

\section{REFERENCES}

546

(1) Rodnina, M. V. Translation in Prokaryotes. Cold Spring Harbor 547 Perspect. Biol. 2018, 10, No. a032664.

(2) Trappl, K.; Mathew, M. A.; Joseph, S. Thermodynamic and 549 Kinetic Insights into Stop Codon Recognition by Release Factor 1. 550 PLoS One 2014, 9, e94058.

(3) Pierson, W. E.; et al. Uniformity of peptide release is maintained 552 by methylation of release factors. Cell Rep. 2016, 17, 11-18.

(4) Zavialov, A. V.; Mora, L.; Buckingham, R. H.; Ehrenberg, M. 554 Release of Peptide Promoted by the GGQ Motif of Class 1 Release 555 Factors Regulates the GTPase Activity of RF3. Mol. Cell 2002, 10, 556 789-798.

(5) Kuhlenkoetter, S.; Wintermeyer, W.; Rodnina, M. V. Different 558 substrate-dependent transition states in the active site of the 559 ribosome. Nature 2011, 476, 351-355.

560

(6) Shoemaker, C. J.; Green, R. Kinetic analysis reveals the ordered 561 coupling of translation termination and ribosome recycling in yeast. 562 Proc. Natl. Acad. Sci. U. S. A. 2011, 108, E1392-E1398.

(7) Petrone, P. M.; Snow, C. D.; Lucent, D.; Pande, V. S. Side-chain 564 recognition and gating in the ribosome exit tunnel. Proc. Natl. Acad. 565 Sci. U. S. A. 2008, 105, 16549-16554.

(8) Murakami, A.; Nakatogawa, H.; Ito, K. Translation arrest of 567 SecM is essential for the basal and regulated expression of SecA. Proc. 568 Natl. Acad. Sci. U. S. A. 2004, 101, 12330-12335.

(9) Gumbart, J.; Schreiner, E.; Wilson, D. N.; Beckmann, R.; 570 Schulten, K. Mechanisms of SecM-mediated stalling in the ribosome. 571 Biophys. J. 2012, 103, 331-341.

(10) Lu, J.; Deutsch, C. Electrostatics in the Ribosomal Tunnel 573 Modulate Chain Elongation Rates. J. Mol. Biol. 2008, 384, 73-86. 574

(11) Ciryam, P.; Morimoto, R. I.; Vendruscolo, M.; Dobson, C. M.; 575 O'Brien, E. P. In vivo translation rates can substantially delay the 576 cotranslational folding of the Escherichia coli cytosolic proteome. 577 Proc. Natl. Acad. Sci. U. S. A. 2013, 110, E132-E140. 578

(12) Frishman, D.; Argos, P. Knowledge-based protein secondary 579 structure assignment. Proteins: Struct., Funct., Genet. 1995, 23, 566- 580 579.

(13) Berman, H. M.; et al. The Protein Data Bank. Nucleic Acids Res. 582 2000, 28, 235-242.

(14) Brooks, B. R.; et al. CHARMM: The Biomolecular Simulation 584 Program. J. Comput. Chem. 2009, 30, 1545-1614. 585

(15) Humphrey, W.; Dalke, A.; Schulten, K. VMD: Visual molecular 586 dynamics. J. Mol. Graphics 1996, 14, 33-38.

(16) Frishman, D.; Argos, P. Incorporation of non-local interactions 588 in protein secondary structure prediction from the amino acid 589 sequence. Protein Eng., Des. Sel. 1996, 9, 133-142.

(17) Dosztanyi, Z.; Csizmok, V.; Tompa, P.; Simon, I. IUPred: web 591 server for the prediction of intrinsically unstructured regions of 592 proteins based on estimated energy content. Bioinformatics 2005, 21, 593 $3433-3434$ 
595 (18) Jorgensen, W. L.; et al. Comparison of simple potential 596 functions for simulating liquid water. J. Chem. Phys. 1983, 79, 926597935.

598 (19) Sillitoe, I.; et al. New functional families (FunFams) in CATH 599 to improve the mapping of conserved functional sites to $3 \mathrm{D}$ 600 structures. Nucleic Acids Res. 2013, 41, D490-D498.

601 (20) Karanicolas, J.; Brooks, C. The origins of asymmetry in the 602 folding transition states of protein L and protein G. Protein Sci. 2002, $60311,2351-2361$.

604 (21) Best, R. B.; Chen, Y. G.; Hummer, G. Slow protein 605 conformational dynamics from multiple experimental structures: 606 The helix/sheet transition of Arc repressor. Structure 2005, 13, 607 1755-1763.

608 (22) O’Brien, E. P.; Christodoulou, J.; Vendruscolo, M.; Dobson, C. $609 \mathrm{M}$. Trigger factor slows Co-translational folding through kinetic 610 trapping while sterically protecting the nascent chain from aberrant 611 cytosolic interactions. J. Am. Chem. Soc. 2012, 134, 10920-10932.

612 (23) Betancourt, M. R.; Thirumalai, D. Pair potentials for protein 613 folding: Choice of reference states and sensitivity of predicted native 614 states to variations in the interaction schemes. Protein Sci. 1999, 8, $615361-369$.

616 (24) Leininger, S. E.; Trovato, F.; Nissley, D. A.; O’Brien, E. P. 617 Domain topology, stability, and translation speed determine 618 mechanical force generation on the ribosome. Proc. Natl. Acad. Sci. 619 U. S. A. 2019, 116, 5523-5532.

620 (25) Nissley, D. A.; O'Brien, E. P. Structural Origins of FRET621 Observed Nascent Chain Compaction on the Ribosome. J. Phys. 622 Chem. B 2018, 122, 9927-9937.

623 (26) Fluitt, A.; Pienaar, E.; Viljoen, H. Ribosome kinetics and aa624 tRNA competition determine rate and fidelity of peptide synthesis. 625 Comput. Biol. Chem. 2007, 31, 335-346.

626 (27) O’Brien, E. P.; Ziv, G.; Haran, G.; Brooks, B. R.; Thirumalai, D. 627 Effects of denaturants and osmolytes on proteins are accurately 628 predicted by the molecular transfer model. Proc. Natl. Acad. Sci. U. S. 629 A. 2008, 105, 13403-13408.

630 (28) Moore, B. L.; Kelley, L. A.; Barber, J.; Murray, J. W.; 631 Macdonald, J. T. High-Quality Protein Backbone Reconstruction 632 from Alpha Carbons Using Gaussian Mixture Models. J. Comput. 633 Chem. 2013, 34, 1881-1889.

634 (29) Rotkiewicz, P.; Skolnick, J. Fast Procedure for Reconstruction 635 of Full-Atom Protein Models from Reduced Representations. J. 636 Comput. Chem. 2008, 29, 1460.

637 (30) Tsui, V.; Case, D. A. Theory and Applications of the 638 Generalized Born Solvation Model in Macromolecular Simulations. 639 Biopolymers 2000, 56, 275-291.

640 (31) Abraham, M. J.; Murtola, T.; Schulz, R.; Pall, S.; Smith, J. C.; 641 Hess, B.; Lindahl, E.; et al. GROMACS: High performance molecular 642 simulations through multi-level parallelism from laptops to super643 computers. SoftwareX 2015, 1-2, 19-25.

644 (32) Hornak, V.; et al. Comparison of Multiple Amber Force Fields 645 and Development of Improved Protein Backbone Parameters. 646 Proteins: Struct., Funct., Genet. 2006, 65, 712-725.

647 (33) Darden, T.; York, D.; Pedersen, L. Particle mesh Ewald: An $648 \operatorname{Nlog}(\mathrm{N})$ method for Ewald sums in large systems. J. Chem. Phys. 649 1993, 98, 10089.

650 (34) Nosé, S. A unified formulation of the constant temperature 651 molecular dynamics. J. Chem. Phys. 1984, 81, 511-519.

652 (35) Nosé, S.; Klein, M. L. Constant pressure molecular dynamics 653 for molecular systems. Mol. Phys. 1983, 50, 1055-1076.

654 (36) Parrinello, M.; Rahman, A. Polymorphic transitions in single 655 crystals: A new molecular dynamics method. J. Appl. Phys. 1981, 52, $6567182-7190$.

657 (37) Hess, B.; Bekker, H.; Berendsen, H. J. C.; Fraaije, J. G. E. M. 658 LINCS: A Linear Constraint Solver for Molecular Simulations. J. 659 Comput. Chem. 1997, 18, 1463-1472.

660 (38) Fritch, B.; et al. Origins of the Mechanochemical Coupling of 661 Peptide Bond Formation to Protein Synthesis. J. Am. Chem. Soc. 2018, $662140,5077-5087$.
(39) O’Brien, E. P.; Vendruscolo, M.; Dobson, C. M. Prediction of 663 variable translation rate effects on cotranslational protein folding. Nat. 664 Commun. 2012, 3, 868.

(40) Mohammad, F.; Green, R.; Buskirk, A. R. A systematically- 666 revised ribosome profiling method for bacteria reveals pauses at 667 single-codon resolution. eLife 2019, 8, 1-25.

(41) Ingolia, N. T.; Ghaemmaghami, S.; Newman, J. R. S.; 669 Weissman, J. S. Genome-Wide Analysis of in Vivo Translation with 670 Nucleotide Resolution Using Ribosome Profiling. Science (Wash- 671 ington, DC, U. S.) 2009, 324, 218-224.

(42) Kamenova, I.; et al. Co-translational assembly of mammalian 673 nuclear multisubunit complexes. Nat. Commun. 2019, 10, 1740.674

(43) Shiber, A.; et al. Cotranslational assembly of protein complexes 675 in eukaryotes revealed by ribosome profiling. Nature 2018, 561, 268. 676 (44) Natan, E.; Wells, J. N.; Teichmann, S. A.; Marsh, J. A. 677 Regulation, evolution and consequences of cotranslational protein 678 complex assembly. Curr. Opin. Struct. Biol. 2017, 42, 90-97. 